Pflegehilfsmittel

Pflege direkt ins Haus

— Die PflegeBox ist Deutschlands führender Komplettservice für die häusliche Versorgung mit Pflegehilfsmitteln. So haben beispielsweise Eltern oder Schwiegereltern, die von Ihren Angehörigen gepflegt werden, ein Anrecht auf Unterstützung. Laut $\S$ 40 SGB XI übernimmt die Pflegekasse beim Vorliegen einer Pflegestufe auf Antrag die Kosten für Pflegehilfsmittel zum Verbrauch von bis zu 40 Euro im Monat. Das sind Produkte wie Einmalhandschuhe, Flächendesinfektion, Händedesinfektion, Bettschutzeinlagen, Schutzschürzen und Mundschutz. Das PflegeBox-Team übernimmt dabei alles Weitere: Von der Beantragung, über die Lieferung bis hin zur Direktabrechnung bei der Pflegekasse.

www.pflegebox.de

PFLEGEBOX

\title{
Diabetestherapie Verbesserung des Glukosestoffwechsels
}

\begin{abstract}
- Basisinsulin ist ein Grundbaustein der Diabetestherapie. Wenn beim Typ 2 die Insulintherapie mit einer basal unterstützten oralen Therapie (BOT) beginnt, hat dies meist eine deutliche Verbesserung des Glukosestoffwechsels zur Folge. Allerdings sollte das Basalinsulin so titriert werden, dass ein normaler Nüchtern-Blutzucker von $<100 \mathrm{mg} / \mathrm{dl}$ bzw. $<5,6 \mathrm{mmol} / \mathrm{l}$ erreicht wird, erklärte der Diabetologe Prof. Thomas Forst aus Neuss auf einer Pressekonferenz in Berlin. Denn eine gute Einstellung entlastet die Betazellen und gewährleistet eine gute Insulinantwort nach den Mahlzeiten. Die Norm-nahe Einstellung der Nüchtern-Glukose setzt voraus, dass das Basalinsulin gleichmäßig über 24 Stunden wirkt und dass diese Wirkung konstant einsetzt. Dies ist bei NPH-Insulinen nicht der Fall, so Forst. Die relativ instabile Absorptionskinetik sorgt für schwankende Basisinsulinspiegel über den Tag, von Tag zu Tag und von Patient zu Patient. Arzt und Patient müssen entweder häufige Unterzuckerungen oder eine nicht so strikte Blutzuckereinstellung
\end{abstract} in Kauf nehmen.

Langwirksame Analoginsuline wie Insulin Glargin oder Insulin Detemir weisen im
Vergleich zu NPH ein flacheres Wirkprofil im 24-Stundenverlauf auf. Mit innen gelingt bei gleich guter Blutzuckerkontrolle eine signifikante Reduktion des Hypoglykämie-Risikos. Einen weiteren Fortschritt ermöglicht eine veränderte Formulierung von Insulin Glargin (U100), die als U300 (Toujeo ${ }^{\circledR}$ ) seit dem Frühjahr 2015 verfügbar ist. Bei U300 liegt das Insulin 3 fach konzentriert vor, was mit einer Halbierung der Absorptionsoberfläche einhergeht. Die Folgen: Längere Halbwertszeit, geringere Absorptionsvariabilität und eine gleichmäßigere Wirkung über 24 Stunden, so Forst. In Vergleichsstudien mit Insulin Glargin zeigte U300 bei gleich guter Blutzuckereinstellung ein signifikant reduziertes Risiko für Hypoglykämien: Über 24 Stunden war es um 14\% reduziert, nachts um $31 \%$. Gerade die Reduktion des nächtlichen Unterzuckerungsrisikos ist laut Forst bedeutsam, denn diese behindern eine erfolgreiche Titration.

(de)

Pressekonferenz "Highlights Sanofi Diabetes 2015: Mit der richtigen Einstellung die Ziele erreichen", Berlin, 4.12.2015 (Veranstalter: Sanofi-Aventis Deutschland $\mathrm{GmbH}$ )

\section{Schallwellenmassage}

\section{Demenzkranken mit Klängen und Musik Körpergefühl vermitteln}

— Basale Stimulation gehört zum Pflegekonzept vieler Einrichtungen, um die neuronalen Verknüpfungen zwischen Wahrnehmung, Bewegung und Kommunikation zu nutzen, um Wohlbefinden und Sicherheit zu vermitteln sowie den Krankheitsverlauf zu verzögern. Neben der Integration des Konzepts in die pflegerische Arbeit am Bewohner wird in vielen Einrichtungen Snoezelen $^{\circledR}$ meist in extra ausgestatteten Räumen angeboten. Manche Einrichtungen stellen fest, dass der Transport der Bewohner aufwändig ist und diese mit der ungewohnten Situation nicht zurecht kommen. Die Demenzkranken können so nicht an Erfahrungen aus der realen Welt anknüpfen. Eine preiswerte Alternative oder Ergänzung, die auf dem Zimmer der Bewohner, in gewohnter Umgebung durchgeführt werden kann, ist die Schallwellenmassage. Neben sensorischer Ansprache bietet sie auch therapeutische Effekte. Mit musik- und toninduzierten Vibrationen durch Schalldruck wird eine sensible Ganzkörperstimulation bewirkt. Mechanorezeptoren in der Haut, in der Muskulatur, in Gelenken und inneren Organen werden sanft stimuliert. Musiktherapeutisch wird das Gehirn parallel auf sensorischer, kognitiver und emotionaler Ebene angesprochen.

Acht Jahre Anwendungserfahrungen deuten darauf hin, dass unter anderem folgende Effekte erreicht werden können: Linderung von Schmerzen des muskuloskeletta- len Systems, Emotionale Stabilisierung, Verlängerung der Aufmerksamkeitsphasen, Intensivere Selbstwahrnehmung, Vegetative Stabilisierung, Muskelentspannung, Lösung von Spasmen, Regulation von Darmund Blasenfunktion, Reduzierung psychomotorischer Unruhe, Lösung depressiver Verstimmungen und die Verbesserung des Ein- und Durchschlafverhaltens.

Die Anwendungen sind bei den Bewohnern sehr beliebt, weil sie den Organismus nicht belasten und als sehr angenehm empfunden werden. Durch die körperliche Entspannung von Patienten, die in ihrer Mobilität eingeschränkt sind, werden viele Handgriffe für das Pflegepersonal leichter. Das Gerät bindet nur geringe Personalkapazität in der Pflege, da es leicht zu bedienen ist und die Bewohner nicht entkleidet werden müssen. Außerdem ist das Gerät mobil und praktisch.

www.schallwellenmassage.de 